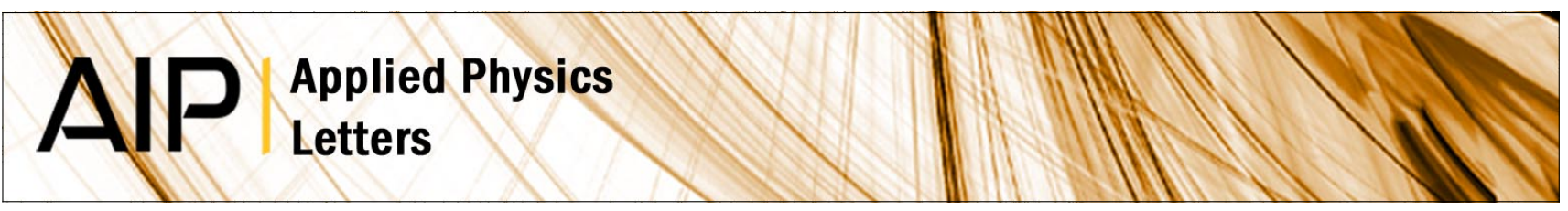

\title{
Highly p-doped epitaxial graphene obtained by fluorine intercalation
}

Andrew L. Walter, Ki-Joon Jeon, Aaron Bostwick, Florian Speck, Markus Ostler et al.

Citation: Appl. Phys. Lett. 98, 184102 (2011); doi: 10.1063/1.3586256

View online: http://dx.doi.org/10.1063/1.3586256

View Table of Contents: http://apl.aip.org/resource/1/APPLAB/v98/i18

Published by the American Institute of Physics.

\section{Related Articles}

Nanopores in $\mathrm{GaN}$ by electrochemical anodization in hydrofluoric acid: Formation and mechanism J. Appl. Phys. 112, 064303 (2012)

Metal nanoantenna plasmon resonance lineshape modification by semiconductor surface native oxide J. Appl. Phys. 112, 044315 (2012)

Asymmetric interfacial abruptness in N-polar and Ga-polar GaN/AIN/GaN heterostructures

Appl. Phys. Lett. 101, 091601 (2012)

On the mechanisms of energy transfer between quantum well and quantum dashes

J. Appl. Phys. 112, 033520 (2012)

Enhanced internal quantum efficiency in graphene/lnGaN multiple-quantum-well hybrid structures

Appl. Phys. Lett. 101, 061905 (2012)

\section{Additional information on Appl. Phys. Lett.}

Journal Homepage: http://apl.aip.org/

Journal Information: http://apl.aip.org/about/about_the_journal

Top downloads: http://apl.aip.org/features/most_downloaded

Information for Authors: http://apl.aip.org/authors

\section{ADVERTISEMENT}
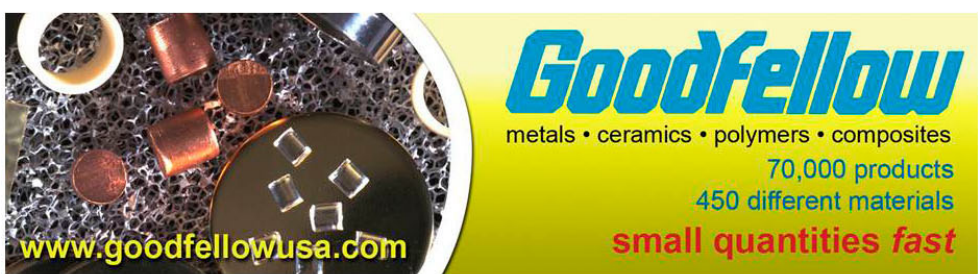


\title{
Highly $p$-doped epitaxial graphene obtained by fluorine intercalation
}

\author{
Andrew L. Walter, ${ }^{1,2, a)}$ Ki-Joon Jeon, ${ }^{3}$ Aaron Bostwick, ${ }^{1}$ Florian Speck, ${ }^{4}$ Markus Ostler, ${ }^{4}$ \\ Thomas Seyller, ${ }^{4}$ Luca Moreschini, ${ }^{1}$ Yong Su Kim, ${ }^{1,5}$ Young Jun Chang, ${ }^{1,2}$ \\ Karsten Horn, ${ }^{2}$ and Eli Rotenberg ${ }^{1}$ \\ ${ }^{1}$ Advanced Light Source (ALS), E. O. Lawrence Berkeley National Laboratory, Berkeley, \\ California 94720, USA \\ ${ }^{2}$ Department of Molecular Physics, Fritz-Haber-Institut der Max-Planck-Gesellschaft, Faradayweg 4-6, \\ 14195 Berlin, Germany \\ ${ }^{3}$ School of Electrical Engineering, University of Ulsan, Namgu, Ulsan 680-749, Republic of Korea \\ ${ }^{4}$ Lehrstuhl für Technische Physik, Universität Erlangen-Nürnberg, Erwin-Rommel-Strasse 1, \\ 91058 Erlangen, Germany \\ ${ }^{5}$ Department of Applied Physics, Hanyang University, Ansan, Gyeonggi-do 426-791, Republic of Korea
}

(Received 10 February 2011; accepted 12 April 2011; published online 3 May 2011)

\begin{abstract}
We present a method for decoupling epitaxial graphene grown on $\mathrm{SiC}(0001)$ by intercalation of a layer of fluorine at the interface. The fluorine atoms do not enter into a covalent bond with graphene but rather saturate the substrate $\mathrm{Si}$ bonds. This configuration of the fluorine atoms induces a remarkably large hole density of $p \approx 4.5 \times 10^{13} \mathrm{~cm}^{-2}$, equivalent to the location of the Fermi level at $0.79 \mathrm{eV}$ above the Dirac point $E_{D}$. (C) 2011 American Institute of Physics.
\end{abstract}

[doi:10.1063/1.3586256]

Many applications of graphene, the single layer of hexagonally coordinated carbon atoms have been proposed since the discovery of its unusual electronic properties. ${ }^{1}$ For these applications to be realized, the ability to achieve $n$ - and $p$-type doping is required. While $n$-type doping is observed in graphene grown epitaxially on $\mathrm{SiC}^{2,3}$ only marginal $p$-type doping has been achieved by hydrogen ${ }^{4-7}$ and $\mathrm{Au}$ (Ref. 8) intercalation or the adsorption of fluorine-containing molecules such as F4-TCNQ. ${ }^{9}$ Here we present a technique for producing highly $p$-doped graphene on $\mathrm{SiC}$ via fluorine intercalation. In contrast to the previous work on fluorinated graphene, (theoretical ${ }^{10}$ and experimental ${ }^{11-14}$ ), where the fluorine is bound to the graphene transforming it into an insulator, here the graphene retains the linear Dirac dispersion that leads to many of its unusual properties.

Previously, hydrogen, ${ }^{4-7}$ and gold ${ }^{8}$ have been shown to intercalate between layers of graphene and $\mathrm{SiC}(0001)$ produced by thermal decomposition. In both cases a slight $p$-doping of the graphene is observed, $p \approx 6 \times 10^{12} \mathrm{~cm}^{-2}$ and $p \approx 7 \times 10^{11} \mathrm{~cm}^{-2}$, respectively. Surface treatment with strongly oxidizing molecules such as $\mathrm{NO}_{2}$ has yielded a large net $p$-doping change around $2 \times 10^{13} \mathrm{~cm}^{-2},{ }^{15}$ but these molecules are not thermally stable, and it is not clear how to protect the molecules from exposure to ambient conditions. In the present study we have used core level x-ray photoemission spectroscopy (XPS) and angle resolved photoelectron spectroscopy (ARPES) to investigate the interaction of fluorine atoms with the $(6 \sqrt{3} \times 6 \sqrt{3}) \mathrm{R} 30^{\circ} / \mathrm{SiC}$ structure and to quantify the amount of $p$ doping. Properly treated, graphene becomes doped to an extremely high level ( $p$ $\approx 4.5 \times 10^{13} \mathrm{~cm}^{-2}$ ) and is stable in air and at high temperatures.

Experiments were performed on $(6 \sqrt{3} \times 6 \sqrt{3}) \mathrm{R} 30^{\circ}$ carbon "buffer layer" on a silicon terminated $\mathrm{SiC}(0001)$ surface, prepared by the method of Ostler et al. ${ }^{16}$ The carbon buffer layer is a covalently bond, electrically inactive graphene

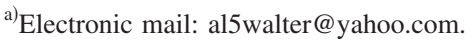

layer ${ }^{17}$ in which about one third of the $\mathrm{C}$ atoms bind to the $\mathrm{Si}$ atoms on the $\mathrm{SiC}(0001)$ substrate. This bonding breaks up the $\pi$ band network so that the $(6 \sqrt{3} \times 6 \sqrt{3}) \mathrm{R} 30{ }^{\circ} \mathrm{C}$ layer (henceforth buffer layer) has no Dirac cone. Hydrogen and gold have been shown to intercalate underneath the buffer layer, breaking the carbon bonds to the substrate and thus decoupling it from the $\mathrm{SiC}$ and transforming it into graphene. ${ }^{4-7}$ Samples were fluorinated following a similar method to McFeely et al. ${ }^{18}$ whereby buffer layer samples are placed in a reaction chamber with a $\mathrm{XeF}_{2}$ crystal and heated to $200{ }^{\circ} \mathrm{C}$ for $2 \mathrm{~h}$, leading to the dissociation of the $\mathrm{XeF}_{2}$ and the liberation of reactive fluorine atoms. This method has previously been used to study the interaction between fluorine and silicon surfaces ${ }^{18}$ as well as fluorine and graphene $\mathrm{e}^{11-14}$ and normally leads to the formation of $\mathrm{C}-\mathrm{F}$ bonds with the graphene layer. If a sacrificial piece of molybdenum is included in the reaction chamber, an entirely different reaction is promoted, whereby the $\mathrm{F}$ atoms bond not to the buffer layer $\mathrm{C}$ atoms, but instead to the interfacial $\mathrm{Si}$ atoms, as discussed below. At high pressure, $\mathrm{XeF}_{2}$ reacts with the molybdenum to produce $\mathrm{MoF}_{6}$ through the wellknown reactions (depending on whether the Mo is clean or oxidized), ${ }^{19,20}$

$$
\begin{aligned}
& 3 \mathrm{XeF}_{2}+\mathrm{Mo} \rightarrow \mathrm{MoF}_{6}(\mathrm{~g})+3 \mathrm{Xe}(\mathrm{g}), \\
& 6 \mathrm{XeF}_{2}+2 \mathrm{MoO}_{3} \rightarrow 2 \mathrm{MoF}_{6}(\mathrm{~g})+6 \mathrm{Xe}(\mathrm{g})+3 \mathrm{O}_{2}(\mathrm{~g}) .
\end{aligned}
$$

Following the fluorination process, the samples are covered by a layer of Mo (oxidized by air exposure), which is readily removed by sonication in ethanol. The presence of this Mo residue indicates that it is the gaseous $\mathrm{MoF}_{6}$, not $\mathrm{XeF}_{2}$, that interacts with the graphene. Samples were transported through air to the electronic structure factory endstation at beamline 7 of the Advanced Light Source, Lawrence Berkeley National Laboratory and annealed to $\sim 600{ }^{\circ} \mathrm{C}$ in vacuum to clean the surface for ARPES and core level measurements. 


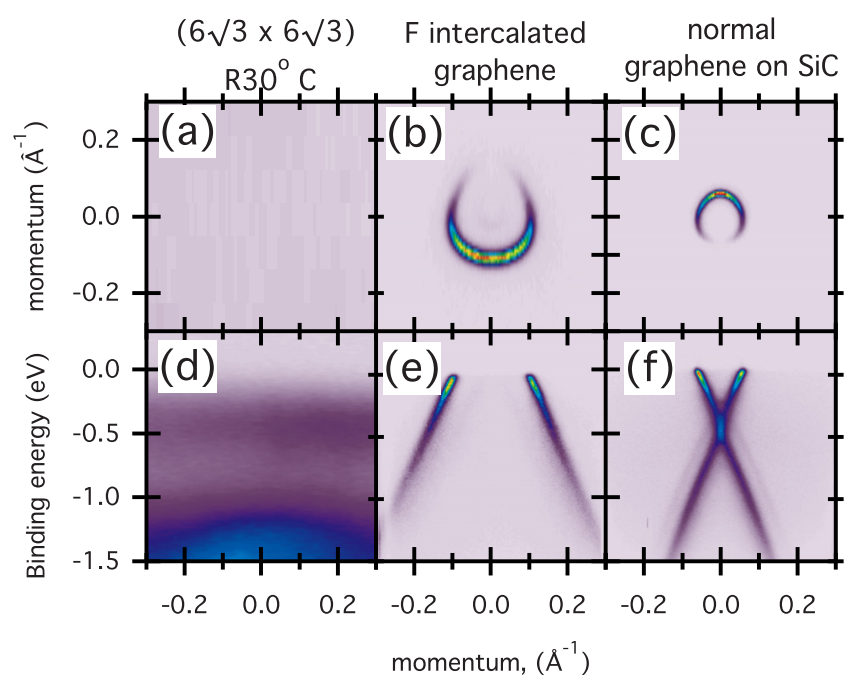

FIG. 1. (Color online) Experimental constant energy cut at the Fermi energy (a, b, and c) and valence band map (d, e, and f) from (a and d) buffer layer, (b and e) graphene prepared by interaction of fluorine with the buffer layer, and ( $c$ and f) graphene on buffer layer. The $p$-doped character of the fluorine intercalated sample is evident.

The Fermi surfaces and band structures of three samples are presented in Figs. 1(a) and 1(d), a buffer-layer sample $(6 \sqrt{3} \times 6 \sqrt{3}) \mathrm{R} 30^{\circ} / \mathrm{SiC}$ without fluorination; Figs. 1(b) and $1(\mathrm{e})$, the same buffer-layer sample later treated with fluorine; and for comparison, Figs. 1(c) and 1(f), a graphene sample grown conventionally, consisting of an active graphene layer on top of the inactive buffer layer. ${ }^{17}$

As reported previously, the buffer layer sample is insulating, ${ }^{7}$ having no Fermi surface and having only a flat band appearing around $0.4 \mathrm{eV}$ binding energy [see Figs. 1(a) and 1(d)]. After fluorination, the circular Fermi surface and linear bands of graphene appear. Projection of the linear bands indicate a Dirac crossing well above the Fermi level, as expected for $p$-type doping, and extrapolating the band to the region above the Fermi edge locates it $0.79 \mathrm{eV}$ above $E_{\mathrm{F}}$. The Fermi surface size can be used ${ }^{21}$ to quantitatively evaluate the hole density at $\left(p \approx 4.5 \times 10^{13} \mathrm{~cm}^{-2}\right)$, which is an order of magnitude larger than previously observed for $\mathrm{H}$ intercalated graphene $\left(p \approx 6 \times 10^{12} \mathrm{~cm}^{-2}\right)$. For comparison, the conventional Argon grown graphene on buffer layer on $\mathrm{SiC}$ samples ${ }^{16}$ are $n$-type with a much smaller charge density $\left(n \approx 1 \times 10^{13} \mathrm{~cm}^{-2}\right)$ and Fermi surface [Figs. 1(c) and 1(f)]. These results suggest that the buffer layer is transformed by fluorination into a $p$-type graphene. The linewidth of the ARPES $\pi$ bands at the Fermi level $\left(\sim 0.014 \AA^{-1}\right)$, which directly gives the electron mean-free path from defect scattering, is comparable to that from the conventional graphene sample $\left(\sim 0.009 \AA^{-1}\right)$ indicating a similar clean, defect-free graphene layer on the fluorine samples. We now discuss core-level photoemission measurements of samples (i) and (ii) which demonstrate that the fluorine intercalates under the buffer-layer/SiC interface. This is completely different from fluorographene/fluorographane materials recently discovered, which are wide band gap semiconductors owing to covalent $\mathrm{C}-\mathrm{F}$ bond formation. ${ }^{13,14,22}$

Core level photoemission spectra of the buffer layer before and after exposure to fluorine are presented in Fig. 2. After fluorine treatment, a clear F $1 s$ peak is observed at $686.9 \mathrm{eV}$ binding energy (not shown), confirming the pres-
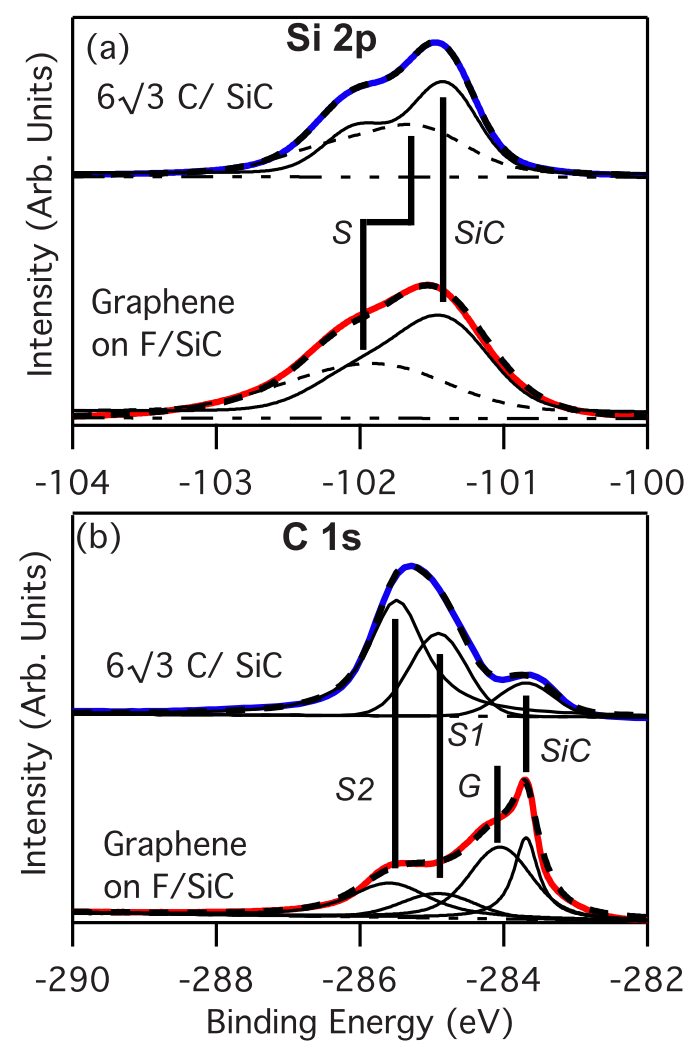

FIG. 2. (Color online) XPS data taken using $350 \mathrm{eV}$ photons of the $(6 \sqrt{3}$ $\times 6 \sqrt{3}) \mathrm{R} 30^{\circ}$ buffer layer before (upper panels) and after (lower panels) fluorination. In each case the thick solid (red and blue) lines represent the experimental data while the dashed and thin solid (black) line represent the fit. (a) Si $2 p$ core levels are fitted to two spin-orbit-split doublet peaks, representing the bulk $(\mathrm{SiC})$ and surface $(S)$ contributions (Ref. 23). Fluorine bonding to interfacial $\mathrm{Si}$ is indicated by a shift in the surface doublet, $S$, of $-0.18 \mathrm{eV}$ after fluorination. (b) C $1 s$ core levels before fluorination consist of a bulk $(\mathrm{SiC})$ peak and two surface peaks $\left(S_{1}, S_{2}\right)$ representing buffer layer $\mathrm{C}$ atoms bonded to the substrate (Ref. 17). After fluorination, the latter peaks are weakened, and a new graphene-derived peak $(G)$ is observed. No C-F bonds are observed.

ence of fluorine. Except for a small residual oxygen peak only peaks relating to $\mathrm{C}, \mathrm{F}$, and $\mathrm{Si}$ are present in XPS scans up to a binding energy of $850 \mathrm{eV}$. The $\mathrm{Si} 2 p$ peak features [Fig. 2(a)] are comparable to previous data from the buffer layer $^{24}$ and are fitted to two doublets representing the bulk $(\mathrm{SiC})$ and surface, $(S)$ contributions to the spectrum. The doublets consist of two Lorentzian-Gaussian peaks with identical line widths separated by $-0.605 \mathrm{eV}$ and with an amplitude ratio of 2:1. In all cases the Lorentzian width is $0.08 \mathrm{eV}$ while both the bulk ( 0.5 to 0.7$)$ and surface $(0.75$ to 1) Gaussian widths increase after fluorination. The increase in the Gaussian width is an indication of inhomogeneity of the sample interface. The position of the bulk doublet is unchanged $(-101.42)$ upon exposure to fluorine, while the surface doublet, $S$, shifts from -101.6 to $-101.78 \mathrm{eV}$, showing that the fluorine interacts with, or is near to, the substrate silicon atoms. This splitting is similar to, but larger than, the Si $2 p$ splitting observed in $\mathrm{H}$-intercalated graphene. ${ }^{4}$ The observed chemical shift is much smaller than observed for $\mathrm{SiF}$ species, ${ }^{18}$ indicating a relatively weak charge transfer from the $\mathrm{Si}$ atoms.

That the fluorine atoms do not covalently bond with the graphene but rather intercalate to the $\mathrm{C}-\mathrm{SiC}$ interface is also evident from C $1 s$ core level spectra shown in Fig. 2(b). The 
top spectrum is from the buffer layer before fluorination and exhibits two peaks $\left(S_{1}, S_{2}\right)$, which arise from the bonding of the carbon atoms in the buffer layer to the $\mathrm{SiC}$ substrate, at $\sim-285.55$ and $-284.75 \mathrm{eV},{ }^{17}$ and a peak at $\sim-283.7 \mathrm{eV}$ due to the bulk SiC. Upon exposure to fluorine, buffer layer peaks $S_{1}, S_{2}$ are largely suppressed, and a new peak $(G)$ at $\sim-284.05 \mathrm{eV}$ appears, attributed to the formation of the graphene layer. Its separation to the carbon peak from the $\mathrm{SiC}$ bulk is much smaller than for graphene on buffer/SiC samples $(\sim-284.74 \mathrm{eV}),{ }^{17}$ which is related to the fact that the graphene is strongly doped. The C $1 s$ spectrum in Fig. 2(b) (lower) also shows none of the $\mathrm{C}-\mathrm{F}$ peaks of perfluorographene ${ }^{12}$ or any peaks in the range 285 to $290 \mathrm{eV}$ expected for $\mathrm{C}-\mathrm{F}$ bonding, ${ }^{12,25}$ for that matter. This provides strong evidence that the fluorine does not bond covalently to the graphene layer. The buffer layer peaks are still present after fluorination, indicating that only partial fluorination of the sample has occurred. Thus the buffer layer is transformed into a single layer of graphene by intercalation, similar to the effect of hydrogen observed in quasifreestanding samples. ${ }^{4,5}$ Similar results (not shown) on thicker initial films have shown that the fluorine interacts with monolayers and bilayers of graphene on buffer layer/SiC, to produce highly $p$-doped bilayer and trilayer graphene, respectively, by converting their buried buffer layers to graphene. Although a detailed interpretation of the binding energy shifts reported above is difficult without considering local field effects, a qualitative picture emerges of an interfacial fluorine layer, chemically bound to $\mathrm{Si}$ atoms, which is fully ionic owing to charge transfer from both the $\mathrm{Si}$ and from the graphene $\mathrm{C}$ atoms. Comparison of the peak areas of the $\mathrm{F} 1 s$ and the C $1 s$ graphene peak [peak $G$ in Fig. 2(b)], and applying the correct photoemission cross-section ratio, ${ }^{26}$ gives (Graphene $\mathrm{C}) / \mathrm{F}$ atomic ratio of $\sim 4.6$. As we have shown that the $\mathrm{F}$ bonds to the surface $\mathrm{Si}$ not the graphene $\mathrm{C}$ then the important ratio is $\mathrm{Si}_{\text {surface }} / \mathrm{F}$ atomic ratio $(\sim 1.7)$, implying a $60 \%$ fluorination of the sample.

In summary, using core and valence band photoemission we demonstrate that fluorine interaction with the carbon buffer layer on $\mathrm{SiC}$ leads to the formation of a strongly $p$-doped graphene layer that is decoupled from the substrate through intercalation, shifting the Dirac crossing point to $0.79 \mathrm{eV}$ above the Fermi energy. This is the strongest $p$-type doping ever observed in graphene, and the fact that the intercalated structure is stable against ambient conditions suggests its use in applications. Moreover, the doping process may be patterned, which could lead to laterally structured $n-p-n$ devices.

The Advanced Light Source is supported by the Director, Office of Science, Office of Basic Energy Sciences, of the U.S. Department of Energy under Contract No. DE-AC02-
05CH11231. Work in Erlangen was supported by the ESF and the DFG through the EUROCORES program EUROGRAPHENE.

${ }^{1}$ A. K. Geim and K. S. Novoselov, Nature Mater. 6, 183 (2007)

${ }^{2}$ A. Bostwick, T. Ohta, T. Seyller, K. Horn, and E. Rotenberg, Nat. Phys. 3, 36 (2007).

${ }^{3}$ K. V. Emtsev, A. Bostwick, K. Horn, J. Jobst, G. L. Kellogg, L. Ley, J. L. McChesney, T. Ohta, S. A. Reshanov, J. Röhrl, E. Rotenberg, A. K Schmid, D. Waldmann, H. B. Weber, and T. Seyller, Nature Mater. 8, 203 (2009).

${ }^{4}$ C. Riedl, C. Coletti, T. Iwasaki, A. A. Zakharov, and U. Starke, Phys. Rev. Lett. 103, 246804 (2009).

${ }^{5}$ F. Speck, M. Ostler, J. Röhrl, J. Jobst, D. Waldmann, M. Hundhausen, L. Ley, H. Weber, and T. Seyller, Mater. Sci. Forum 645-648, 629 (2010).

${ }^{6}$ A. Bostwick, F. Speck, T. Seyller, K. Horn, M. Polini, R. Asgari, A. H. MacDonald, and E. Rotenberg, Science 328, 999 (2010).

${ }^{7}$ C. Virojanadara, R. Yakimova, A. Zakharov, and L. Johansson, J. Phys. D 43, 374010 (2010).

${ }^{8}$ I. Gierz, T. Suzuki, R. T. Weitz, D. S. Lee, B. Krauss, C. Riedl, U. Starke, H. Höchst, J. H. Smet, C. R. Ast, and K. Kern, Phys. Rev. B 81, 235408 (2010).

${ }^{9}$ C. Coletti, C. Riedl, D. S. Lee, B. Krauss, L. Patthey, K. V. Klitzing, J. H. Smet, and U. Starke, Phys. Rev. B 81, 235401 (2010).

${ }^{10}$ D. Boukhvalov and M. Katsnelson, J. Phys.: Condens. Matter 21, 344205 (2009).

${ }^{11}$ F. Withers, M. Dubois, and A. Savchenko, Phys. Rev. B 82, 073403 (2010).

${ }^{12}$ J. T. Robinson, J. S. Burgess, C. E. Junkermeier, S. C. Badescu, T. L. Reinecke, F. K. Perkins, M. K. Zalalutdniov, J. W. Baldwin, J. C. Culbertson, P. E. Sheehan, and E. S. Snow, Nano Lett. 10, 3001 (2010).

${ }^{13}$ R. R. Nair, W. Ren, R. Jalil, I. Riaz, V. G. Kravets, L. Britnell, P. Blake, F. Schedin, A. S. Mayorov, S. Yuan, M. I. Katsnelson, H.-M. Cheng, W. Strupinski, L. G. Bulusheva, A. V. Okotrub, I. V. Grigorieva, A. N. Grigorenko, K. S. Novoselov, and A. K. Geim, Small 6, 2877 (2010).

${ }^{14}$ K.-J. Jeon, Z. Lee, E. Pollak, L. Moreschini, A. Bostwick, C.-M. Park, R. Mendelsberg, V. Radmilovic, R. Kostecki, T. J. Richardson, and E. Rotenberg, ACS Nano 5, 1042 (2011).

${ }^{15}$ S. Zhou, D. Siegel, A. Fedorov, and A. Lanzara, Phys. Rev. Lett. 101, 086402 (2008).

${ }^{16}$ M. Ostler, F. Speck, M. Gick, and T. Seyller, Phys. Status Solidi B 247, 2924 (2010)

${ }^{17}$ K. Emtsev, F. Speck, T. Seyller, L. Ley, and J. Riley, Phys. Rev. B 77, 155303 (2008).

${ }^{18}$ F. McFeely, J. Morar, N. Shinn, G. Landgren, and F. Himpsel, Phys. Rev. B 30, 764 (1984)

${ }^{19}$ J. Veyan, D. Aureau, Y. Gogte, P. Campbell, X. M. Yan, and Y. J. Chabal, J. Appl. Phys. 108, 114913 (2010).

${ }^{20}$ R. Burns, I. D. MacLeod, T. A. O’Donnell, T. E. Peel, K. A. Phillips, and A. Waugh, J. Inorg. Nucl. Chem. 39, 1737 (1977).

${ }^{21}$ T. Ohta, A. Bostwick, T. Seyller, K. Horn, and E. Rotenberg, Science 313, 951 (2006).

${ }^{22}$ H. Peelaers, A. D. Hernandez-Nieves, O. Leenaerts, B. Partoens, and F. M. Peeters, Appl. Phys. Lett. 98, 051914 (2011).

${ }^{23}$ J. Carlisle, T. Miller, and T.-C. Chiang, Phys. Rev. B 49, 13600 (1994).

${ }^{24}$ T. Seyller, K. Emtsev, K. Gao, F. Speck, L. Ley, A. Tadich, L. Broekman, J. Riley, R. Leckey, O. Rader, A. Varykhalov, and A. Shikin, Surf. Sci. 600, 3906 (2006)

${ }^{25}$ Y. Sato, K. Itoh, R. Hagiwara, T. Fukunaga, and Y. Ito, Carbon 42, 3243 (2004)

${ }^{26}$ J. J. Yeh and I. Lindau, At. Data Nucl. Data Tables 32, 1 (1985). 